Recibido:

junio 22 de 2021

Aceptado:

octubre 27 de 2021

\section{Gestión del talento humano en el Centro Clínico Los Ángeles, C. A.}

Management of human talent at Centro Clínico Los Ángeles, C. A.

\author{
Irvana del Carmen, Villarroel Díaz ${ }^{\mathbf{1}}$ \\ irvanav@gmail.com \\ https://orcid.org/0000-0002-9164-2564
}

\author{
Sandra J. Estrada \\ sandraestrada92@gmail.com
ORCID: 0000-0003-3440-0224 \\ sandraestrada92@gmail.com \\ https://doi.org/10.22209/amr.v1n1a05
}

\section{Resumen}

La presente investigación estuvo dirigida a analizar la gestión del talento humano en el Centro Clínico Los Ángeles, C. A., basándose teóricamente en Zabaleta (2013), Chiavenato (2009), Vallejo (2016), Dolan y Cabrera (2007), Calderón (2008), entre otros. La investigación fue de tipo analítica, con diseño no experimental, transversal y de campo; en la cual se aplicó como técnica de recolección de datos la entrevista estructurada y el instrumento de guía de entrevista. La validez fue de contenido mediante juicio de expertos. Los datos se analizaron a través de una matriz de análisis, permitiendo concluir que efectivamente en la empresa en estudio se aplican los procesos de gestión de talento humano tales como: admisión, aplicación, compensación, desarrollo, mantenimiento y monitoreo de personas, sin embargo, se pudo constatar que la empresa no basa su gestión de recursos humanos en los modelos de gestión de conocimiento y por competencias, siendo ello una oportunidad de mejora para potencializar su gestión estratégica. En cuanto a las tendencias, sus directrices se enfocan en la búsqueda del posicionamiento en el mercado mediante una filosofía de gestión de calidad en sus servicios.

Palabras clave: calidad organizacional; control interno; gestión empresarial; recursos humanos.

1 Facultad de Ciencias Administrativas, Universidad Alonso de Ojeda, Venezuela. 


\begin{abstract}
This research study aimed at analyzing management of human talent at Centro Clínico Los Ángeles, C. A., based on the theoretical considerations from Zabaleta (2013), Chiavenato (2009), Vallejo (2016), Dolan and Cabrera (2007), Calderón (2008), among other authors. This was an analytical research study with a non-experimental, cross-sectional field design. A structured interview and an interview guide were applied as data collection techniques. Content validity was established through expert judgement. The data was analyzed through an analysis matrix, which led to conclude that talent management processes are indeed applied in the company under study, including: admission, application, compensation, development, retention and monitoring of employees. However, it was found that the human resource management of the company is not based on knowledge and competency management models, this being an opportunity for improvement to enhance its strategic management. Regarding the talent management trends applied in the clinical center, its guidelines focus on the search for market positioning through a philosophy of quality management in its services.
\end{abstract}

Keywords: organizational quality; internal control; business management; human resources.

\title{
Introducción
}

Debido a los avances tecnológicos y a las nuevas necesidades del sector productivo tanto a nivel local como internacional, en la actualidad, las empresas deben asumir el compromiso de gerenciar de manera eficaz el talento humano que contribuye en el constante progreso de sus actividades, tomando en cuenta que los trabajadores para poder satisfacer sus necesidades básicas y cumplir con sus objetivos individuales, dependen en la mayoría de los casos de las organizaciones en las cuales prestan sus servicios; es por ello, que la relación empresa - colaborador debe ser concebida dentro del principio del equipo de trabajo a través del cual se promueva la sinergia corporativa, por cuanto en la medida en que un trabajador pueda ver alcanzado sus objetivos, éste contribuirá de manera significativa en la producción sus bienes y servicios de calidad y consecuentemente, en el alcance los objetivos estratégicos corporativos.

Es así como los colaboradores en las organizaciones representan algo más que un simple recurso, sino, un talento humano que genera valor agregado en la consecución de los objetivos empresariales, lo cual debe motivar a la gerencia contemporánea a emprender acciones que le permita gestionar de forma 
efectiva, la selección, retención y desarrollo de sus trabajadores, siendo que ellos, mediante la interpretación de la misión corporativa y ejecución de actividades concretas, son parte esencial para la empresa; le dan vida, aportan al desarrollo y contribuyen a su sostenibilidad. En este sentido, Dolan y Cabrera (2007), consideran que la gestión de talento humano consiste en desarrollar y poner en práctica las políticas y programas que permitan generar oportunidades de progreso para los empleados.

De igual manera, Vallejo (2016) menciona que el proceso de reclutamiento, capacitación y desarrollo del personal necesario para la conformación de grupos de trabajo de calidad, toma su tiempo, por lo cual, las empresas deben tomar conciencia sobre lo importante que es conservar el capital humano, aunque ello no es una tarea muy sencilla, debido a que en algunos casos priman los intereses y/o necesidades del propio colaborador frente a situaciones que son ajenas a la unidad de negocios.

Aun así, hay una realidad que cobija a las organizaciones del momento. Cuando se trata de pérdidas en materia de capital, maquinarias o equipos dentro de una empresa, se considera que puede existir una solución a través de la cobertura de una prima de seguros, algún préstamo u otra alternativa que puede contribuir a recuperar o reponer estos recursos materiales; sin embargo, para el caso de la fuga de talento humano, esas vías de solución no siempre son posibles de implementar en el tiempo requerido por la propia organización, por cuanto se necesita de una buena labor y en algunos casos, el empleo de alta suma de dinero para recuperarlo; no obstante, como se indicó en el párrafo anterior, se está frente a una tarea que no es sencilla debido a que cada persona es un mundo sujeto a la influencia de distintas variables, de aptitudes propias al ser humano y a patrones de comportamientos muy diversos.

Dado lo anterior, esta investigación tuvo como objetivo analizar la gestión del talento humano en el Centro Clínico Los Ángeles, C.A. Para la realización de este trabajo se tomaron en cuenta aspectos como el actual funcionamiento del área de recursos humanos; los procesos que se cumplen al momento de reclutar, seleccionar y retener al personal en la empresa; con la finalidad de identificar cuáles son los factores más resaltantes que debilitan el desarrollo a largo plazo de los colaboradores y de esta manera, poder tomar medidas preventivas para la consolidación de un grupo de recursos humanos ampliamente idóneo para el alcance de la misión corporativa. 


\section{Fundamentación teórica}

Para todas las empresas en general, la gestión del recurso humano puede significar la clave del éxito y la esencia de la gerencia organizacional. Con una excelente gestión, la empresa puede crear una mejor calidad de trabajo y prestación de servicios, para que sus colaboradores se sientan incentivados a ejecutar sus funciones contribuyendo significativamente en el alcance de la misión corporativa. La gestión del talento humano es sin duda alguna, una realidad que afecta a todas las áreas de una organización, por lo que la gerencia debe enfocarse en potenciar las habilidades y conocimientos de sus trabajadores de forma cónsona con las actividades a realizar en los planes de trabajo.

En este orden de ideas, la gestión del talento humano como tal, se implementa en los años 90 cuando David Watkins utiliza el término para representar el proceso que cumple toda empresa con el fin de atraer y retener trabajadores productivos que sumen valor a la organización; para esta época, se produce el auge del término de talento humano, puesto que las personas se preparaban para ser expertos en el ámbito laboral y las organizaciones visualizaban los beneficios de trabajar con una correcta gestión del talento organizacional basada en personas con altas competencias para el logro efectivo de sus labores y el mayor rendimiento organizacional.

Por su parte, Chiavenato (2009), plantea que la gestión del talento humano es utilizada por las empresas inteligentes; las que son capaces de comprender que lo que promueve el éxito empresarial a largo plazo, es el talento, habilidades y destrezas de sus empleados, sin embargo, la realidad que hoy día caracteriza el mundo laboral no es del todo fácil, al contrario, algunas veces es común encontrar trabajadores que no están de acuerdo o satisfechos con el oficio que desempeñan, la remuneración y el ambiente laboral, convirtiéndose ello en una preocupación para muchos gerentes, teniendo en consideración los cambios que ocurren en las fuerzas de trabajos y las pérdidas que le ocasionan a la organización tanto a nivel corporativo como económico, debido a la rotación constante y la salida de personal de alto valor para las empresas.

A su vez, Caldera (2007), considera que es responsabilidad de las empresas gestionar de manera efectiva el talento que posee, tomando en cuenta que una correcta gestión implica la captación, capacitación, desarrollo, conservación del talento humano. Cabe destacar, que las teorías que definen la gestión del personal, coinciden en que se trata de identificar al empleado como el principal protagonista de las organizaciones, reconociendo sus atributos y habilidades individuales en lo que se refiere al rol que desempeña, sus conocimientos, 
competencias y hasta la propia relación que puede tener con el resto de los trabajadores como base a una idónea gestión.

Ahora bien, en el contexto latinoamericano, la consultora Deloitte (2018) publicó un artículo titulado "Gestión RRHH en América Latina" en donde después de realizar diferentes estudios en varios países, se obtuvo como resultado que uno de los grandes problemas que genera gran tensión para las organizaciones, es el entorno cultural mixto, donde conviven valores nacionales y regionales, al igual que prácticas globales y locales. En el caso de Venezuela, expone Amador (2003) que existen pocas organizaciones que han entendido la relación valor-conocimiento como base fundamental para la conceptualización del capital humano y su importancia para el desarrollo empresarial, pero aquellas que han implementado la gestión del talento ha involucrado una cantidad importante de recursos para capacitar de forma continua a quienes conforman su equipo de trabajo, sobre todo, tomando en consideración el valor de éstos frente al alcance de la filosofía de gestión.

En ese sentido, Espinoza (2014) publica un artículo titulado "Gerencia de Recursos Humanos en Venezuela", en el cual hace referencia al cambio significativo que ha tenido en las últimas décadas la gerencia administrativa, específicamente en el área de recursos humanos; pasando de una situación de abundancia económica a un ambiente caracterizado por la escasez de recursos, creciente complejidad y acentuado dinamismo. De la misma manera, Chirinos (2011), señala que con el pasar de los años, se ha incrementado de manera significativa el cierre masivo de empresas privadas, por lo cual, resalta la gran cantidad de pequeñas y medianas empresas que laboran sin un área o departamento enfocado en la gestión del recurso humano; por otra parte, las empresas privadas que aún mantienen sus operaciones activas, enfocan principalmente sus esfuerzos en atraer personal que cumpla con las tareas necesarias para el desarrollo de sus actividades pero que al mismo tiempo, puedan sentirse en condiciones físicas y emocionales para realizar adecuadamente su labor.

Lo antes señalado, trae como consecuencia una rotación constante en los puestos, inflexibilidad con los horarios de trabajo e incluso una sobre carga de responsabilidades no establecidas al momento de la contratación, constituyéndose ello en una situación de incomodidad laboral para los colaboradores; provocando incertidumbre con respecto a las metas profesionales y en la competitividad de las compensaciones recibidas, descenso del rendimiento, inflexibilidad laboral $y$, en el peor de los casos, la salida no prevista del talento humano. 


\section{Metodología}

En lo que se refiere al tipo de investigación, el presente trabajo se tipifica como analítico, puesto que pretendió describir la gestión del talento humano en el Centro Clínico Los Ángeles. Al respecto, Hurtado (2000) define este tipo de investigación como aquel método que pretende obtener pruebas y validar una información o razonamiento a través de mecanismo verificables y de la observación de fenómenos, revelando características fundamentales del tema estudiado. De igual manera esta investigación tuvo un diseño no experimental, debido a que en la misma no se construyó situación ninguna; solo se observaron realidades ya existentes sin ser manipuladas por los investigadores. En este mismo orden de ideas, el trabajo se enmarca dentro de los diseños transversales, basándose en el hecho de que los datos fueron recolectados en un único momento de campo.

En cuanto a la población objeto de estudio, la misma estuvo conformada por 85 trabajadores del Centro Clínico Los Ángeles, C. A.

Tabla 1. Distribución Poblacional

\begin{tabular}{|l|c|}
\hline \multicolumn{1}{|c|}{ Departamentos } & Numero de Población \\
\hline Asamblea de Socios & 3 \\
\hline Administrativo & 16 \\
\hline Financiero & 5 \\
\hline Operativo Medico & 56 \\
\hline Seguridad & 5 \\
\hline Total & 85 \\
\hline
\end{tabular}

Fuente: elaboración propia, 2020

Con relación a la muestra, esta estuvo conformada por la Gerente General del Departamento de Recursos Humanos del Centro Clínico Los Ángeles, C. A. En cuanto a la técnica de recolección de datos se empleó la entrevista, la cual es definida por Fernández (2016) como aquella herramienta que permite una comunicación fluida, natural y espontánea en las que el sujeto informante expone sus conocimientos de acuerdo a sus experiencias o estudios obtenidos, permitiéndole así al investigador recopilar toda la información necesaria la cual será base fundamental para la obtención de resultados y su posterior análisis. Por su parte Cornejo y Salas (2011) indican que la entrevista debe generar profundidad e interrelación de los acontecimientos, sentimientos y relatos expuestos por el informante. 
En ese sentido, para llevar a cabo la recolección de datos a través de la entrevista, fue necesario la implementación de un instrumento el cual permitió desarrollar de manera eficaz esta técnica, siendo éste la guía de entrevista, definida por Atehortúa y Zwerg (2012) como aquela herramienta metodológica que contiene las interrogantes y aspectos que se desean analizar en una entrevista; basada principalmente en preguntas abiertas las cuales permitirán que el investigador aclare cada uno de los puntos relevantes para su estudio, mediante el ejercicio de una conversación natural y fluida con el informante.

En el orden del proceso metodológico, la validez del instrumento de recolección de datos fue de contenido mediante juicio de 3 expertos quienes certificaron su eficacia y pertinencia de cada una de las preguntas formuladas y su correspondencia con la variable en estudio. Como técnica de interpretación de resultados se aplicó el análisis cualitativo. Para tal fin, se organizó la información obtenida en una matriz de resultados que le permitió a los investigadores examinar la información y obtener los datos más relevantes a partir de la adecuada comprensión de los mismos frente al propósito de analizar la gestión del talento humano en el Centro Clínico Los Ángeles, C. A.

\section{Resultados}

En este segmento se presentan los resultados obtenidos a través de la aplicación de la entrevista como técnica de recolección de datos, expuestos en el orden de presentación de la variable gestión del talento humano, sus dimensiones e indicadores. Se presenta a continuación una tabla resumen de los hallazgos por cada dimensión:

Tabla 2. Resumen de Hallazgos: Procesos de Gestión del Talento Humano.

\begin{tabular}{|l|c|l|}
\hline \multicolumn{1}{|c|}{ DIMENSIÓN } & INDICADOR & \multicolumn{1}{c|}{ HALLAZGOS } \\
\hline Procesos de \\
$\begin{array}{l}\text { Gestión de Talento } \\
\text { Humano }\end{array}$ & $\begin{array}{l}\text { La recepción y/o reclutamiento del personal se realiza a través de: } \\
\text { Recomendaciones personales. }\end{array}$ \\
$\begin{array}{l}\text { Recón } \\
\text { Contratación de pasantes que anteriormente formaron parte } \\
\text { del personal de la empresa y que cumplen las características } \\
\text { requeridas para los puestos de trabajos. } \\
\text { Para seleccionar el personal, se necesita: } \\
\text { Realización de entrevista con el departamento de recursos } \\
\text { humanos. } \\
\text { Entrevista entre el jefe del área donde se presenta la vacante y el } \\
\text { aspirante al cargo. }\end{array}$ \\
\hline
\end{tabular}




\begin{tabular}{|c|c|c|}
\hline DIMENSIÓN & INDICADOR & HALLAZGOS \\
\hline \multirow{5}{*}{$\begin{array}{l}\text { Procesos de } \\
\text { Gestión de Talento } \\
\text { Humano }\end{array}$} & Aplicación & $\begin{array}{l}\text { Al incluir nuevos colaboradores en la empresa, se realiza lo } \\
\text { siguiente: } \\
\text { - Inducción los aspectos generales de la empresa y las áreas o } \\
\text { - } \text { departamentos. } \\
\text { Facilitación de herramientas y elementos necesarios para realizar } \\
\text { las labores. } \\
\text { Presentación del trabajador ante el jefe inmediato, supervisores y } \\
\text { - } \quad \text { Rempañeros de trabajo. } \\
\text { - Se proporciona información sobre las políticas y normas a cumplir } \\
\text { en la empresa. }\end{array}$ \\
\hline & Compensación & $\begin{array}{ll}\text { Las compensaciones que se otorgan son: } \\
\text { - } \quad \text { Incentivos monetarios. } \\
\text { - } \quad \text { Asistencia médica gratuita. }\end{array}$ \\
\hline & Desarrollo & $\begin{array}{l}\text { De acuerdo a la obtención de conocimientos por parte de los } \\
\text { colaboradores, se realiza: } \\
\text { - Ejecución de charlas y talleres cada tres (3) meses según los } \\
\text { requerimientos del desarrollo del personal. }\end{array}$ \\
\hline & Mantenimiento & $\begin{array}{l}\text { Se ofrecen los siguientes atractivos: } \\
\text { Para el personal en general: } \\
\text { - } \quad \text { Buen clima organizacional y estabilidad laboral. } \\
\text { - } \quad \text { Aire acondicionado, sillas y escritorios ergonómicos } \\
\text { - } \quad \text { Equipos de seguridad y computación. } \\
\text { Para el personal médico: } \\
\text { - Consultorios dotados con los materiales necesarios para los } \\
\quad \text { procedimientos médicos } \\
\text { - } \quad \text { Quirófanos, rayos X, entre otros. } \\
\text { - Personal de apoyo }\end{array}$ \\
\hline & Monitoreo & $\begin{array}{l}\text { Se aplican lo siguiente: } \\
\text { De manera directa: } \\
\text { - El supervisor o jefe de área realiza evaluaciones de desempeño } \\
\quad \text { en función de las tareas que se delegan. } \\
\text { De manera indirecta: } \\
\text { - Vigilancia mediante sistema de cámaras de seguridad distribuidas } \\
\quad \text { por área. } \\
\text { La información de los empleados se almacena de forma física en } \\
\text { carpetas tipo expediente con la siguiente información: copia del } \\
\text { documento de identidad, copia del título de formación académica, } \\
\text { referencias laborales, el contrato de trabajo y los recibos de pago y/o } \\
\text { préstamos otorgados. }\end{array}$ \\
\hline
\end{tabular}

Fuente: elaboración propia, 2020. 
Tabla 3. Resumen de Hallazgos: Modelos de Gestión del Talento Humano.

\begin{tabular}{|c|c|l|}
\hline \multicolumn{1}{|c|}{ DIMENSIÓN } & INDICADOR & \multicolumn{1}{c|}{ HALLAZGOS } \\
\hline \multirow{2}{*}{$\begin{array}{l}\text { Modelos de } \\
\text { Gestión del Talento } \\
\text { Humano }\end{array}$} & $\begin{array}{l}\text { Lestión del } \\
\text { Conocimiento } \\
\text { mismos de aportar conocimientos, ideas de trabajo y acciones de } \\
\text { mejora continua en las actividades bajo su responsabilidad. Resaltando } \\
\text { la postura del área de recursos humanos de empresa en estudio, al } \\
\text { afirmar que los trabajadores deben aportar conocimientos sin contar } \\
\text { con estrategias que fomenten dicha actividad, es decir, la empresa } \\
\text { no cumple con un modelo de gestión basado en la utilización y } \\
\text { desarrollo del conocimiento. }\end{array}$ \\
\cline { 2 - 4 } & $\begin{array}{l}\text { Los empleados se ven en la necesidad de ser conscientes por sí } \\
\text { mismos de aportar conocimientos, ideas de trabajo y acciones de } \\
\text { mejora continua en las actividades bajo su responsabilidad. Resaltando } \\
\text { la postura del área de recursos humanos de empresa en estudio, al } \\
\text { afirmar que los trabajadores deben aportar conocimientos sin contar } \\
\text { con estrategias que fomenten dicha actividad, es decir, la empresa } \\
\text { no cumple con un modelo de gestión basado en la utilización y } \\
\text { desarrollo del conocimiento. }\end{array}$ \\
\hline
\end{tabular}

Fuente: elaboración propia, 2020.

Tabla 4. Resumen de Hallazgos. Tendencias de Gestión del Talento Humano.

\begin{tabular}{|c|c|l|}
\hline DIMENSIÓN & INDICADOR & \multicolumn{1}{c|}{ HALLAZGOS } \\
\hline \multirow{4}{*}{$\begin{array}{l}\text { Tendencias de } \\
\text { Gestión del Talento } \\
\text { Humano }\end{array}$} & $\begin{array}{c}\text { Proyección } \\
\text { organizacional }\end{array}$ & $\begin{array}{l}\text { Dirección de la gerencia frente a los esfuerzos de los trabajadores } \\
\text { en pro de posicionar la organización como principal centro de } \\
\text { salud privado de la región. }\end{array}$ \\
\cline { 2 - 3 } & Gestión del cambio del & Fomento de cambios en los procesos de realización de labores. \\
\cline { 2 - 3 } & Gestión de calidad & $\begin{array}{l}\text { Desempeño del liderazgo por parte de los jefes de unidades } \\
\text { departamentales. }\end{array}$ \\
\cline { 2 - 3 } & Productividad & $\begin{array}{l}\text { Existencia de normas y políticas de calidad como parte del Sistema } \\
\text { de Control Interno. }\end{array}$ \\
\hline $\begin{array}{l}\text { Trabajo en función de cumplir con cada uno de los procesos y } \\
\text { lineamientos que les permitan alcanzar el nivel de productividad. }\end{array}$ \\
\hline
\end{tabular}

Fuente: elaboración propia, 2020.

\section{Discusión}

Con relación a la dimensión procesos de gestión del talento humano, se puede afirmar que los resultados coinciden con lo que señalan autores como Dolan y Cabrera (2007) y Chiavenato (2009). Éste último plantea que la gestión moderna de talento humano, presenta los siguientes procesos: reclutamiento del personal, aplicación, compensación, laboral, desarrollo y retención del personal, los cuales 
se aplican formalmente en la empresa objeto de estudio. Al respecto se evidenció que la gerencia de la empresa realiza el proceso de reclutamiento basado inicialmente en recomendaciones de terceros, ello, aunque coincide con la postura de Torres-Flórez et al. (2020), los cuales refieren que es una propuesta idónea, la selección de recursos humanos mediante de terceros interesados en los objetivos de la organización tal es el caso de los propios trabajadores y el personal directivo, por cuanto éstos conocen las necesidades e intereses de la organización, y al mismo tiempo, tienen la oportunidad de sugerir personas que cumplan con competencias humanas que estén alineadas a los principios y valores éticos de la empresa; aun así, es opinión de los investigadores que la empresa debe mirar hacia convocatorias públicas donde cumpliendo los adecuados filtros y retroalimentación en el proceso de reclutamiento, pueda tener diferentes alternativas para la selección del talento humano, sobre todo, en beneficio de los objetivos organizacionales.

En el caso de la dimensión modelos de gestión del talento humano, se puede señalar que los resultados obtenidos son totalmente opuestos a lo que señalan autores como Valera y Bedoya (2006) y Louffat (2005), para quienes los modelos de gestión del talento humano, son considerados como un soporte para la administración moderna, siendo éstos los de conocimientos y competencias. Los mencionados autores afirman que ambos modelos son esenciales en la nueva filosofía empresarial basada en la valorización de las personas. Por tanto, aunque en la institución objeto de estudio se apliquen las fases del proceso de gestión del talento humano tradicionalmente aplicadas, al no emplear un modelo para esa gestión, quedan sin considerarse aspectos de gran importancia para la valorización del personal y, por ende, necesarios para la optimización de procesos y el alcance de resultados corporativos.

Finalmente, con relación a las tendencias de gestión del talento humano, las cuales se aplican en la institución escogida para este estudio, puede señalarse que los resultados son cónsonos con lo que afirman varios autores, entre los cuales se destaca Calderón (2008), quien señala que en la actualidad, y en relación a esta temática, las tendencias en la gestión del recurso humano y las prácticas que visibilizan esta corriente, tienen el propósito aumentar la productividad y competitividad organizacional.

Por su parte, Matabajoy-Montilla et al. (2018), exponen que, dentro de la tendencia en la gestión del talento humano, recobra mayor fuerza además de cumplir con los procesos naturales de selección y contratación, la capacitación, calidad de vida laboral y evaluación de desempeño. En su investigación, Matabajoy-Montilla et al. (2018) refieren la importancia de que la gerencia de recursos humanos 
emprenda acciones que se orienten a la detección de necesidades y creación de programas de capacitación que sean cónsonos con los objetivos de la empresa y que, a su vez, contribuyan a la productividad y sostenibilidad empresarial.

\section{Conclusiones}

En virtud de los resultados obtenidos en esta investigación, desde la caracterización del problema de estudio, su sustentación teórica, el enfoque metodológico y el respectivo análisis de los resultados, se establecen las siguientes conclusiones: con relación a la primera dimensión, procesos de gestión del talento humano utilizados en el Centro Clínico Los Ángeles, C. A., se pudo determinar que efectivamente se cumple un proceso de gestión del talento humano tradicional y consecuentemente, ajustado a criterios de control interno en la materia sujeta a análisis. Las etapas establecidas en el proceso de gestión humana son la de admisión, aplicación, compensación, desarrollo, mantenimiento y monitoreo de personas.

En lo que se refiere a la admisión de personas, se realizan actividades como reclutar a través de recomendaciones personales realizadas por otros trabajadores de la empresa o directamente contratar a personas que estén realizando pasantías o a personas que anteriormente hayan pasado por el proceso de reclutamiento y selección, pero en el momento no cumplían con los requisitos necesarios. Aún así, es importante resaltar las nuevas tendencias de aplicar procesos de selección de personal mediante convocatorias públicas que permitan a la gerencia, tener mayor amplitud en las opciones frente a los objetivos de la organización.

En cuanto a la aplicación de personas, el departamento de recursos humanos de la empresa dirige sus esfuerzos en realizar en el menor tiempo posible el reclutamiento y selección del personal, enfocándose en la integración de los nuevos colaboradores y dándoles todos los detalles que necesiten como conocer las áreas, los directivos, supervisores y compañeros, así como también las herramientas y equipos de trabajo que tendrán a su cargo; del mismo modo, se le suministra cualquier tipo de información que sea necesaria para la ejecución eficaz en sus funciones en cumplimiento del sistema de control interno.

Útil es mencionar que, en relación con la compensación de personas, esta organización ofrece a los colaboradores que integran todas las áreas de trabajo, incentivos del tipo monetario aparte de servicios médicos de emergencia para el empleado y facilidad de pago en caso de ser familiar directo. Así mismo, en función del desarrollo de personas, se realizan charlas y talleres cada tres meses, 
lo cual es sumamente necesario para capacitar a los trabajadores en temas de interés general para la ejecución de sus labores. Con relación al mantenimiento de personas, se debe agregar que se ofrecen como atractivos un buen clima de trabajo y seguridad laboral. En cuanto al monitoreo de personas, éste se realiza a través de la observación indirecta por parte de los supervisores en una evaluación de desempeño mensual y de manera directa, mediante sistemas de vigilancia con cámaras de seguridad.

Seguidamente con respecto a la dimensión, modelos de gestión del talento humano en el Centro Clínico Los Ángeles, C. A, es trascendental señalar que la organización de estudio no se ocupa por establecer una inclinación directa por alguno de los modelos de gestión del talento humano, dando por hecho que los empleados que integran la empresa deben realizar sus labores aplicando los conocimientos o técnicas que posean como habilidades adquiridas, sin basarse en algún modelo que permita orientar sus talentos como recursos que generen un valor en el alcance de los objetivos empresariales.

Para finalizar con la última dimensión relacionada a las tendencias de gestión del talento humano que se aplican en el Centro Clínico Los Ángeles, C. A., se llegó a la conclusión de que la proyección organizacional es una de las directrices que se logra alcanzar en función de la manera en que se dirigen las capacidades internas de los recursos humanos, puesto que los esfuerzos se enfocan en lograr los requerimientos del mercado con la finalidad de posicionarse como una de las principales opciones para los habitantes de la ciudad en cuanto a servicios de salud se refiere.

Por lo que se refiere a la gestión del cambio, los resultados demuestran algunas deficiencias internas debido a que la gerencia no aplica estrategias que propicien la comunicación e innovación en la realización de labores, de igual manera sucede con el liderazgo de las personas en las diferentes áreas que componen a la organización, por cuanto hasta el momento de la investigación, solo los jefes o supervisores cumplen el papel de líder sin facilitar la oportunidad de desarrollar en los demás colaboradores esta competencia como alternativa de personal relevo.

Atendiendo a las consideraciones anteriormente presentadas, en la organización sujeta a estudio aplican criterios orientados para la gestión mediante el establecimiento de políticas de calidad que promueven el mejoramiento continuo de las técnicas y métodos de trabajo. En cuanto a la productividad, si bien es cierto que se brindan incentivos que podrían motivar a los colaboradores en la ejecución de sus labores y por consiguiente ampliar los resultados obtenidos, en 
la organización se presentan faltas en la formación de sus empleados, además de una infraestructura organizacional no completamente idónea a sus objetivos, lo que conlleva a que los colaboradores sean menos eficientes y no respondan frente a los resultados esperados.

A manera general, queda demostrado que, el Centro Clínico Los Ángeles, C.A., se basa en las etapas que conforman el proceso de gestión donde cada una de ellas se realiza enfocada en atraer, incorporar, compensar, desarrollar, mantener y monitorear a los recursos humanos necesarios para el cumplimiento de las actividades de la organización.

En contraste con lo anterior, se presenta una gestión deficiente en la aplicación de alguno de los modelos de gestión de talento humano que influye directamente en el desarrollo organizacional y ocasionan una mala distribución de esfuerzos entre el personal que labora en la organización, el no enfocar sus esfuerzos en crear un sistema de gestión que les proporcione a los empleados un ambiente laboral orientado hacia un modelo que permita el intercambio de conocimientos o desarrollo de capacidades que favorezca el reconocimiento del valor del talento humano como pieza fundamental en el logro del éxito organizacional.

Finalmente, se concluye que, aunque se reconocen los aspectos positivos en la gestión de talento humano que pone en práctica la gerencia actual, es necesario identificar algunos retos que ésta debe asumir para optimizar sus recursos humanos y contribuir significativamente en el alcance de los resultados corporativos. Estos retos son: generar líneas de acción para crear técnicas que contribuyan promover los conocimientos y habilidades de sus colaboradores y fomentar estrategias que desarrollen el liderazgo individual de cada puesto de trabajo, los colaboradores.

\section{Referencias}

Amador, J. (2003). Proceso Administrativo. http://www.elprisma.com/apuntes/ administracion_de_empresas/procesoadministrativo/

Atehortúa, F. y Zwerg-Villegas, A. (2012). Metodología de la investigación: más que una receta. AD-minister, 20, 91-111.

Caldera, R. (2007). El Recurso humano en las empresas. http://es.wikipedia.org/wiki/ Recursos_humanos.

Calderón, G. (2008). Aproximación a un modelo de gestión humana que agregue valor a la empresa colombiana. Universidad Nacional de Colombia. Colombia.

Cornejo, M. y Salas, N. (2011). Rigor y calidad metodológicos: un reto a la investigación social cualitativa. Psicoperspectivas, 10(2), 12-34. 
Chiavenato, I. (2009) Gestión del Talento Humano. Primera Edición. Editorial Mc GrawHill. Colombia

Chirinos, J. (2011). Perspectiva teórica de la Innovación organizacional y la Gestión del Talento humano en las empresas públicas del Estado Zulia, Venezuela. Revista Reveitec Urbe. Vol. 2. Num. 1. diciembre 2011. http://ojs.urbe.edu/index.php/ revecitec/article/view/1413.

Deloitte. (2018). Gestión de RRHH en América Latina. https://www2.deloitte.com/ content/dam/Deloitte/ec/Documents

/human-capital/Gestion\%20de\%20RRHH\%20en\%20America\%20Latina.pdf.

Dolan, S. y Cabrera, R. (2007) Gestión de Recursos Humanos. Tercera Edición, Editorial Mac Graw Hill. Colombia.

Espinoza, R. (2014). Gerencia de Recursos humanos en Venezuela. erevistas.saber. ula.ve. http://www.saber.ula.ve/bitstream/handle/123456789/25070/articulo7. pdf?sequence $=2 \&$ isAllowed $=y$

Fernández, P. (2016). Acerca de los enfoques cuantitativo y cualitativo en la

Hurtado, J. (2000). Metodología de la investigación holística. https://ayudacontextos. files.wordpress.com/2018/04/jacqueline-hurtado-de-barrera-metodologia-deinvestigacion-holistica.pdf

Investigación educativa cubana actual. Atenas, 34(2), 1-15.

Louffat, E. (2005). Convergencia de los modelos de conocimiento de competencias como soporte para la moderna administración de empresas. Universidad de Sao Paulo. Brasil.

Matabajoy-Montilla, J. M., Matabachoy-Tulcán, S. M., \& Obando-Guerrero, L. M. (2018). Procesos de desarrollo del talento humano en una clínica de especialidades de Pasto, Colombia. Universidad y salud, 20(1), 26-36.

Torres-Flórez, D., Velásquez-Díaz, J. S., \& Hernández-González, J. W. (2020). Importancia del reclutamiento y la selección del personal en el sector hotelero: Caso Villavicencio-Colombia. Desarrollo Gerencial, 12(1), 1-23.

Varela R. y Bedoya, O. (2006). Modelo conceptual de desarrollo empresarial basado en competencias. <http://www.scielo.org.co/scielo.php?script=sci_arttext\&pid=S012359232006000300001\&lng=en\&nrm=iso>. ISSN 0123-5923.

Vallejo, L. (2016). Gestión del talento humano. Primera Edición. Editorial Espoch. Ecuador. 